Check for updates

Cite this: RSC Adv., 2019, 9, 4609

Received 29th November 2018 Accepted 30th January 2019

DOI: $10.1039 / c 8 r a 09818 b$

rsc.li/rsc-advances

\section{Unveiling the thickness-dependent mechanical properties of graphene papers by in situ SEM tension $\uparrow$}

\author{
Tianci Cao, Xianqiang Liu, * Xiaopeng Cheng, Yonghe Li, (D) Lijun Sang, Jinyao Ma, \\ Jin Wang, Jiachuo He, Mingming Wang and Yuefei Zhang (D)
}

\begin{abstract}
With more and more applications, the mechanical strength of graphene paper (GP) has attracted significant attention in recent years. In this report, GPs were prepared by flow-induced filtration of electrochemical exfoliated graphene sheets. By adjusting the concentration of solution, we found graphene sheets fabricated in $0.1 \mathrm{M} \mathrm{K}_{2} \mathrm{SO}_{4}$ have the thinnest average thickness. And by uniaxial in-plane tensile tests operated on a self-developed in situ scanning electron microscopy (SEM) tensile stage, the corresponding GP has the best fracture strength of $192 \mathrm{MPa}$. This is due to that the thickness decrease of exfoliated graphene will increase the quantity of interlayer crosslinks, thus improving the mechanical properties of GPs. This research may open a new way to obtain high-strength GPs for applications.
\end{abstract}

\section{Introduction}

Graphene, a single atom thick $2 \mathrm{D}$ honeycomb $\mathrm{sp}^{2}$ carbon lattice, has been attracting a great deal of attention due to its high specific surface area $\left(2640 \mathrm{~m}^{2} \mathrm{~g}^{-1}\right)$, high flexibility, fine mechanical strength, thermal and chemical stability, and high conductivity ${ }^{1-3}$ since it was experimentally discovered in $2004 .{ }^{4}$ For macroscopical application, as building blocks graphene can be assembled to graphene paper (GP), which has emerged in batteries, ${ }^{5}$ supercapacitors, ${ }^{6}$ sensors,${ }^{7}$ filtration membrane ${ }^{8}$ and so on.

Whether for electrochemical field applications or filtration membrane, mechanical strength is of great importance to GPs. The mechanical properties of prepared GPs have been investigated by tensile, indentation, and bending test. ${ }^{9}$ The high strength and stiffness of GPs were ascribed to the interlockingtile microstructures of graphene nanosheets. ${ }^{10}$ To quantitatively understand the mechanical properties of stratified material, several theoretical models have been proposed. In 1952, Cox put forward the shear-lag model for fibrous materials in consider of the interface shear between hard and soft phases. ${ }^{11}$ After that, Gao and $\mathrm{Li}$ extended this model into carbon nanotube composites. ${ }^{12}$ In 2003, Ji and Gao proposed the tension-shear chain (TSC) model. ${ }^{13}$ Afterwards, this model was further extended to explain the failure mechanism of biological materials with hierarchical microstructures like bone and teeth. In

Institute of Microstructure and Property of Advanced Materials, Beijing University of Technology,Beijing, 100124, PR China.E-mail: xqliu@bjut.edu.cn; yfzhang@bjut. edu.cn

$\dagger$ Electronic supplementary information (ESI) available. See DOI: 10.1039/c8ra09818b
TSC model, the interface shear stress between bone and protein is uniform because of the rigidity of bones. ${ }^{14}$ In contrast, graphene sheets have good elasticity compared to bones. Thus, Liu et al. modified TSC to predict the mechanical properties of graphene-based papers, which is the deformable tension-shear (DTS) model. ${ }^{15}$

From one aspect, the mechanical property of GO paper should be better than that of GP because of the oxygencontaining chemical groups. However, these oxygencontaining groups are harmful to the application of GP in the electrochemical field. Up to now, people have no idea about how to improve the mechanical properties of GP. Here, in this paper, by tuning the electrolyte, we fabricated graphene of different thickness and assembled them into GPs. The combination of in situ tensile testing inside SEM and following AFM analysis found that the fracture strength of GPs has strong correlation with the quality of graphene sheets prepared.

\section{Experiments}

\subsection{Materials}

Graphite foil (99.8\%) was provided by Alfa Aesar chemical Co., Ltd., China. $\mathrm{K}_{2} \mathrm{SO}_{4}$ (AR) was supplied by Zhiyuan Chemical Reagent Co., Ltd., China.

\subsection{Preparation of graphene}

Graphite foil, platinum plate, $0.1 \mathrm{~mol} \mathrm{~L}^{-1}(\mathrm{M}) \mathrm{K}_{2} \mathrm{SO}_{4}$ solution were used for working electrode, counter electrode, and the electrolyte, respectively. The distance between two electrodes was about $3 \mathrm{~cm}$. A constant $10 \mathrm{~V}$ voltage was applied for 15 minutes, and the flow rate was controlled at $\sim 2 \mathrm{~cm} \mathrm{~s}^{-1}$. After 
being vacuum-filtered and washed by deionized water (DIW), the wet GP was redispersed in DIW by sonication for $20 \mathrm{~min}$ and then centrifuged at $2000 \mathrm{rpm}$ for $20 \mathrm{~min}$. The supernate was collected and centrifuged at $4000 \mathrm{rpm}$ for $30 \mathrm{~min}$. The precipitate was collected. These cleaning steps were repeated for 3 times and the preparation of $0.1 \mathrm{M}$ graphene was finished. Other conditions remained invariable, only changing the concentration of $\mathrm{K}_{2} \mathrm{SO}_{4}$ solution then we can get $0.2 \mathrm{M}, 0.3 \mathrm{M}$ and 0.4 $\mathrm{M}$ graphene, respectively.

\subsection{Preparation of graphene paper}

GPs were prepared by vacuum-filtrating the resulting graphene colloid through an Anodic membrane filter $(47 \mathrm{~mm}$ in diameter, $0.2 \mu \mathrm{m}$ pore size, Whatman), and then drying at $80^{\circ} \mathrm{C}$. The thickness of GPs is about $16 \mu \mathrm{m}$.

\subsection{Characterization}

Scanning electron microscopy (SEM) (FEI Quanta 250, accelerating voltage: $20 \mathrm{kV}$ ) was used to observe the morphologies of surface and fracture surface of GPs. The morphologies and structure of graphene were characterized by transmission electron microscopy (TEM) (FEI Titan G2, operated at $300 \mathrm{kV}$ ).
The lateral dimensions and height were measured using the atomic force microscopy (AFM) (Bruker Multimode8, tapping mode). Typical uniaxial in-plane tensile tests were operated on a self-developed in situ specimen tensile stage integrated in Quanta 650 FEG ESEM. ${ }^{16}$ All tensile tests were conducted in controlled strain rate mode ${ }^{17}$ with a preload of $0.01 \mathrm{~N}^{18}$ and a stain ramp rate of $11 \mu \mathrm{m} \mathrm{min}{ }^{-1}$.

\section{Results and discussion}

\subsection{Electrochemical exfoliation and GPs preparation}

Four kinds of graphene (Fig. 1a) were obtained from a twoelectrode system in the electrolyte $\mathrm{K}_{2} \mathrm{SO}_{4}$ solution ${ }^{19}$ prepared by dissolving $\mathrm{K}_{2} \mathrm{SO}_{4}$ in DIW (concentration of 0.1-0.4 M). The mechanism of electrochemical exfoliaton is illustrated in Fig. 1b: Firstly, graphite is intercalated by $\mathrm{OH}^{-}$ions $\left(4 \mathrm{OH}^{-}-\right.$ $4 \mathrm{e}^{-} \rightarrow 2 \mathrm{H}_{2} \mathrm{O}+\mathrm{O}_{2}$ ), which 'opens' the edge and grain boundaries of graphite. The expansion of graphite layers facilitates the following anions intercalation. Finally, reduction of $\mathrm{SO}_{4}{ }^{2-}$ yields a large amounts of $\mathrm{SO}_{2}$ gas $\left(\mathrm{SO}_{4}{ }^{2-}+4 \mathrm{H}^{+}+2 \mathrm{e}^{-} \rightarrow 2 \mathrm{H}_{2} \mathrm{O}+\right.$ $\mathrm{SO}_{2}$ ), supplying force to separate weakly-bonded graphite layers into graphene sheets. The exfoliated graphene sheets (EGs) will (a)

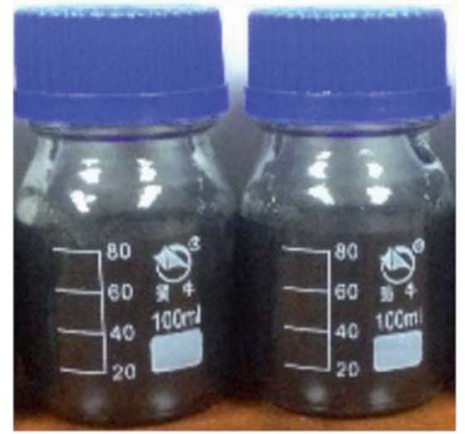

(b)
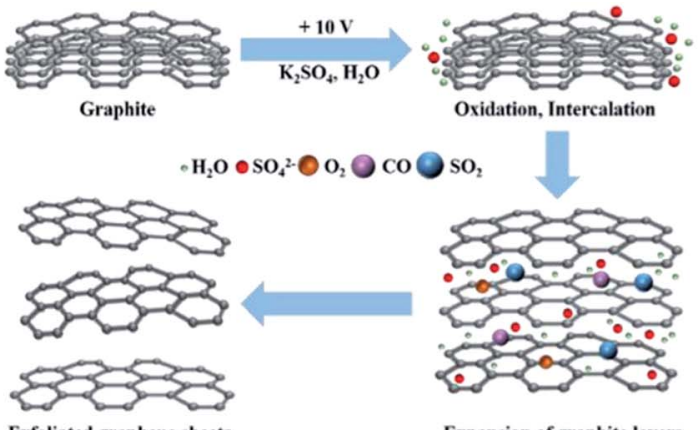

Exfoliated graphene sheets
Oxidation, Intercalation

$\mathrm{SO}_{2}$

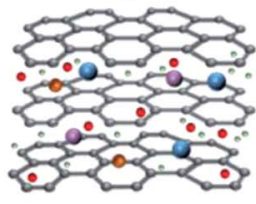

Expansion of graphite layers

GP

(c)

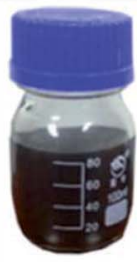

Graphene
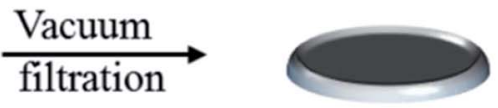

AAO disk
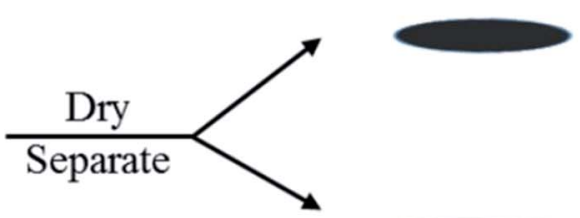

(d)
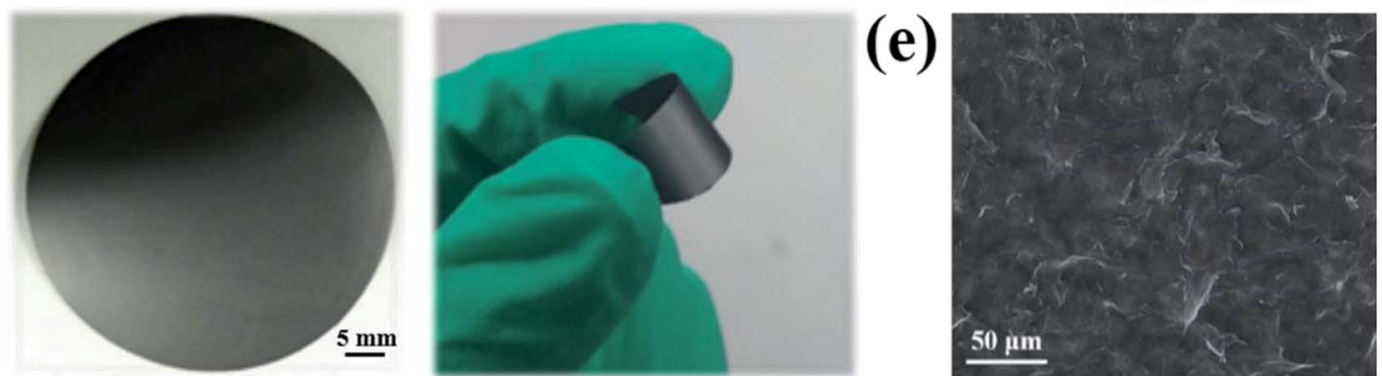

Fig. 1 (a) Dispersed graphene in ethyl alcohol. (b) Schematic illustration of the mechanism of electrochemical exfoliation. (c) Schematic of the fabrication procedure of freestanding GP. (d) Photographs of freestanding GP. (e) SEM image of surface morphology of GP. 
(a)

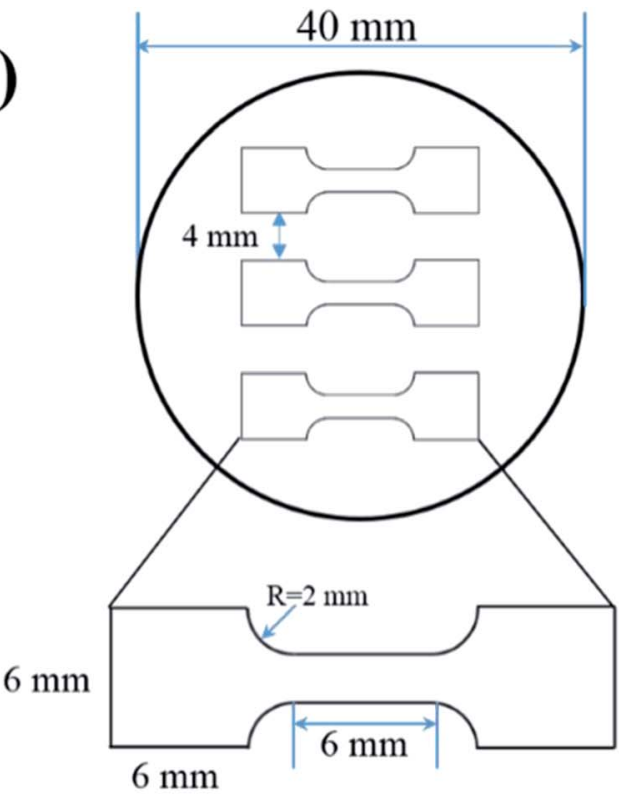

(b)

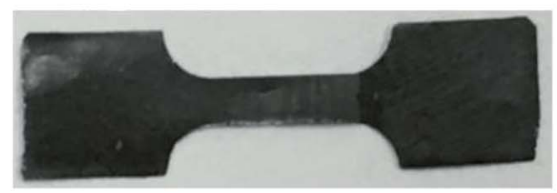

(c)

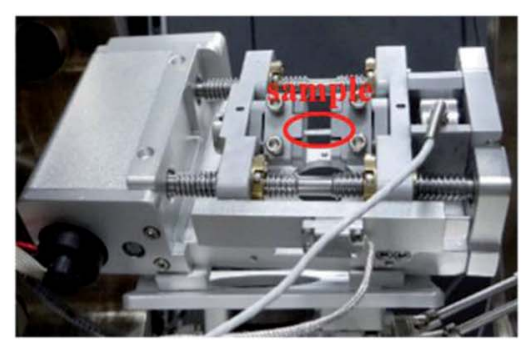

(d)

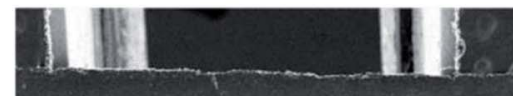

(e)

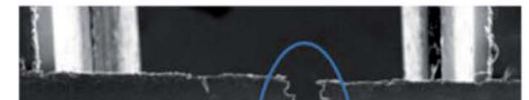

(f)

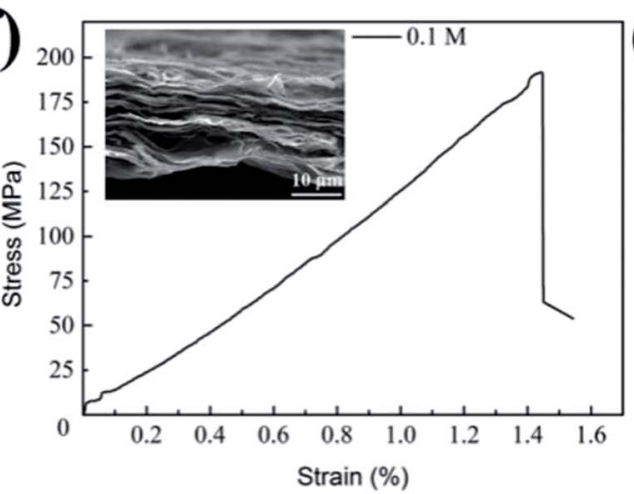

(h)

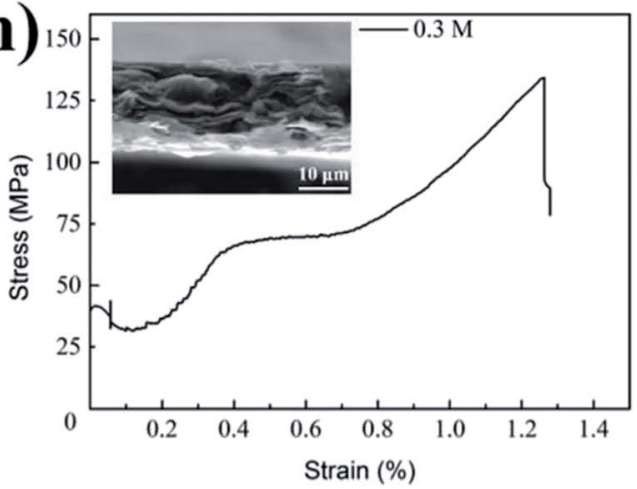

(g)

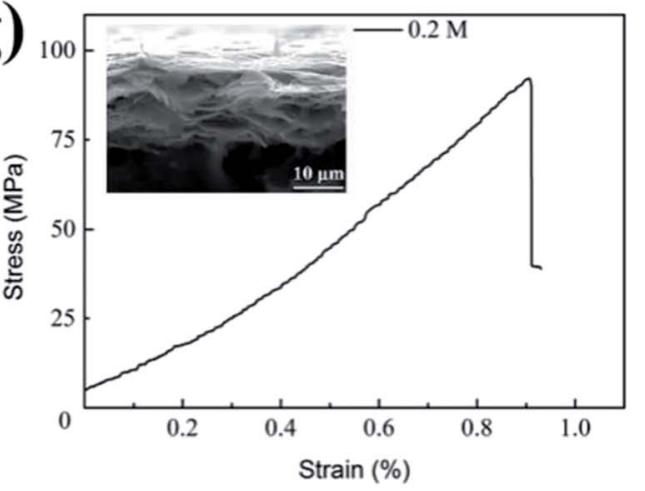

(i)

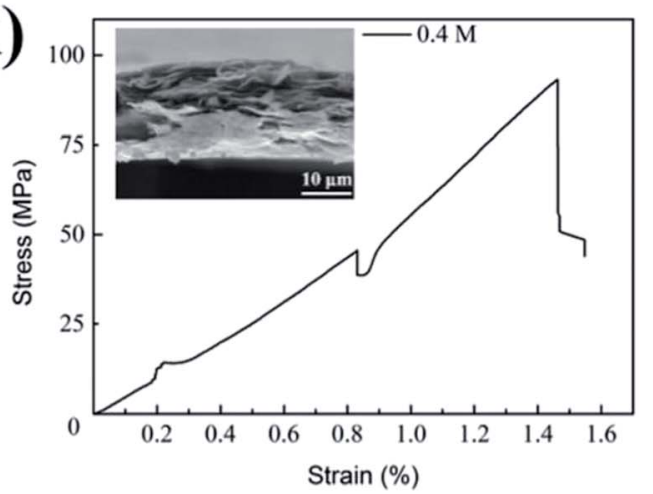

Fig. 2 (a) The blueprint of GP sample. (b) The prepared GP strip. (c) In situ specimen tensile stage integrated in SEM, strip before (d) and after (e) fracture from tensile loading. ( $f-i)$ Stress-strain curves for samples at preparation condition: $0.1 \mathrm{~mol} \mathrm{~L}^{-1}(\mathrm{M}) \mathrm{K}_{2} \mathrm{SO}_{4}, 0.2 \mathrm{M}, 0.3 \mathrm{M}, 0.4 \mathrm{M}$ and the SEM images (insets) of fracture surface morphology. 


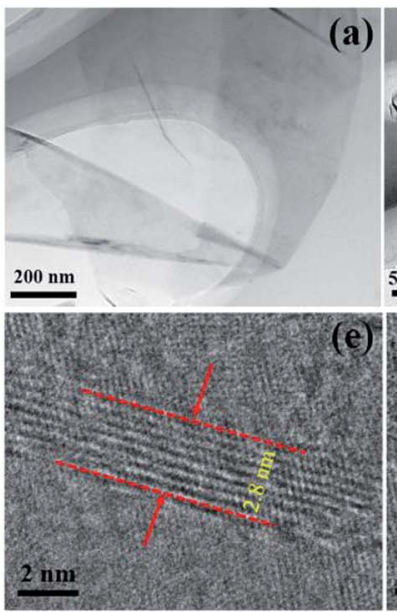

$0.1 \mathrm{M}$

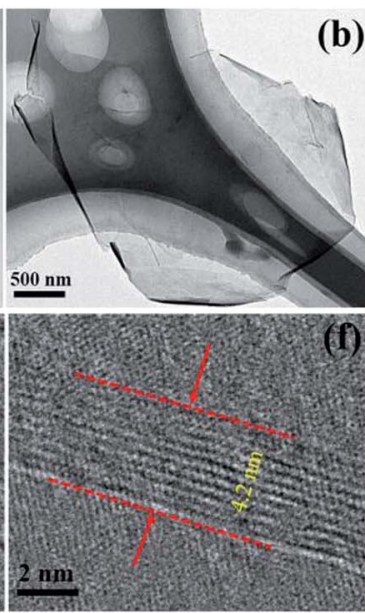

$0.2 \mathrm{M}$

(b)

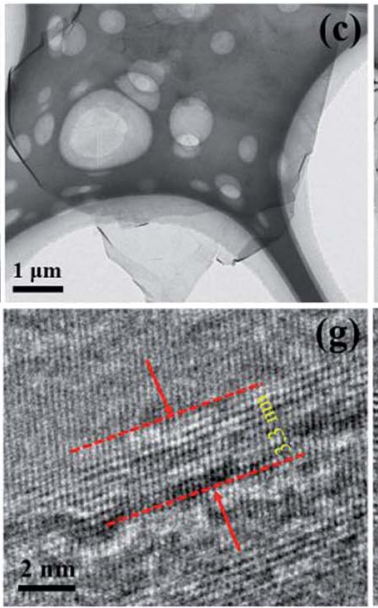

$0.3 \mathrm{M}$

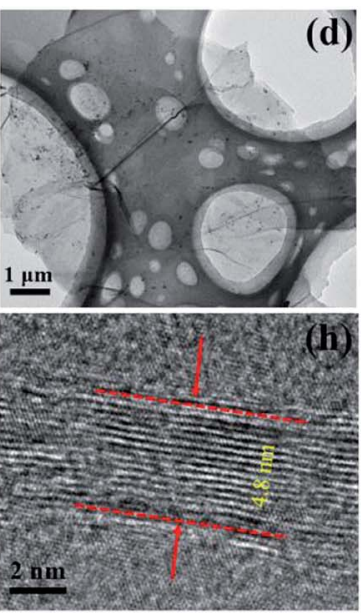

$0.4 \mathrm{M}$

Fig. $3(a-d)$ TEM images of graphene sheets of four conditions: $0.1 \mathrm{M}, 0.2 \mathrm{M}, 0.3 \mathrm{M}, 0.4 \mathrm{M}$. (e-h) HRTEM images of four samples and the thickness of graphene sheets are $2.8,4.2,3.3,4.8 \mathrm{~nm}$, respectively.

contain less oxygen-containing groups compared with the graphene prepared by oxide-reduction methods. Then, the GPs were prepared via a procession of vacuum-filtration, drying and separation (Fig. 1c). The resulted GPs exhibit good flexibility (Fig. 1d). The surface morphology of GPs was characterized by SEM (Fig. 1e), showing the piece-by-piece overlapping hierarchical structure.

\subsection{In situ measurements of mechanical properties of GPs}

Fig. $2 \mathrm{a}$ is the sketch figure of the mould for the preparation GP tensile samples in in situ mechanical measurements. And Fig. $2 \mathrm{~b}$ is the real GP strips for uniaxial in-plane tensile tests. Fig. 2c shows the specimen tensile stage designed for in situ experiments. Fig. $2 \mathrm{~d}$ and e exhibit the low magnification morphologies of GPs before and after tensile experiments, respectively. And the morphology shows out the characterization of brittle fracture. Fig. 2f-i present the stress-strain curves of GPs, corresponding to four kinds of EGs $(0.1,0.2,0.3$ and 0.4 $\mathrm{M})$, respectively. These curves exhibit linear behavior (elastic), similar to the reports of Shen et al. ${ }^{20}$ and Wang et al. ${ }^{21}$ However, the fracture strength of four kinds GPs (f-i) varies dramatically and they are 191.68, 93.16, 134.25 and 92.18 MPa, respectively. The corresponding fracture morphologies (insets) demonstrate obvious laminar structure, suggesting the hierarchical structure of GPs. Interestingly, we found that the strips of $0.1 \mathrm{M}$ and $0.3 \mathrm{M}$ seem to have more sheets than $0.2 \mathrm{M}$ and $0.4 \mathrm{M}$ and correspondingly they have higher fracture strength.

\subsection{Characterization of graphene sheets}

To explore the origin of dramatic variation of fracture strength, we carefully characterized the building blocks of these GPs. Fig. 3a-d show the bright field images of four kinds of EGs, which exhibit the thin flaky and weak wrinkle morphologies. Moreover, the relative brighter characteristics for EGs in Fig. 3a and c suggest a little thinner thickness in comparison with those in Fig. $3 \mathrm{~b}$ and d. Fig. 3e-h present the HRTEM images obtained from the curly edges of these EGs, which will clearly show the thickness of these EGs: they are 2.8, 4.2, 3.3, $4.8 \mathrm{~nm}$, respectively, agreeing well with the characteristics in Fig. 3a-d.

Due to the difficulty of analyzing large amount of samples by TEM, we did the thickness statistics of four kinds of EGs according to the AFM measurements. ${ }^{22}$ Fig. 4 shows the morphologies, height profiles and layer number statistics of four kinds of EGs, respectively. Statistical data show that all four kinds of EGs have monolayer graphene, which is thinner than $1 \mathrm{~nm}$. And over $80 \%$ of EGs have less than 5 layers in all samples. The comparison shows that the electrolyte concentration has a non-negligible influence on the quality of EGs. The samples of electrolyte concentration at $0.1 \mathrm{M}$ have over $30 \%$ monolayer EGs. In comparison, the samples of $0.4 \mathrm{M}$ have less than $10 \%$ monolayer EGs. The average thicknesses of four kinds of EGs are $2.81 \mathrm{~nm}, 3.53 \mathrm{~nm}, 3.05 \mathrm{~nm}, 4.27 \mathrm{~nm}$, respectively. According to the procedure of electrochemical exfoliaton, $\mathrm{OH}^{-}$ ions provide gaps between graphite layers and $\mathrm{SO}_{4}{ }^{2-}$ ions offer force to separate layers from these gaps. Therefore, the number of gaps determines the thickness of the prepared graphene. When concentration of solution increases, the formation of $\mathrm{OH}^{-}$ions is suppressed due to the low water content, besides, due to the high concentration of $\mathrm{SO}_{4}{ }^{2-}$ ions, the exfoliation process will finish rapidly before the formation of new gaps. From the above, higher electrolyte concentration corresponds to thicker graphene sheets thickness within a certain concentration range. Thus a low concentration electrolyte will produce EGs of high quality, as shown by Fig. 4 .

\subsection{Analysis of mechanical property}

Fig. 5a sketches the uniaxial in-plane tension of GP strips. As shown by Fig. 5a, GPs are formed by the stacking and 
[ I ]

(a)

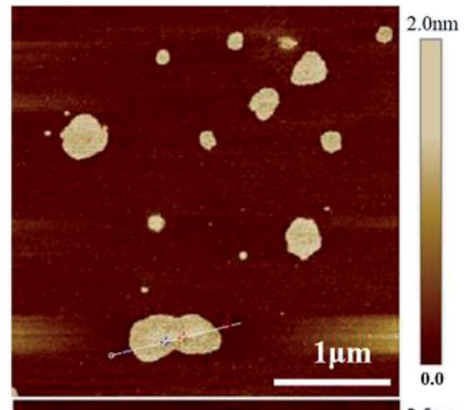

(b)

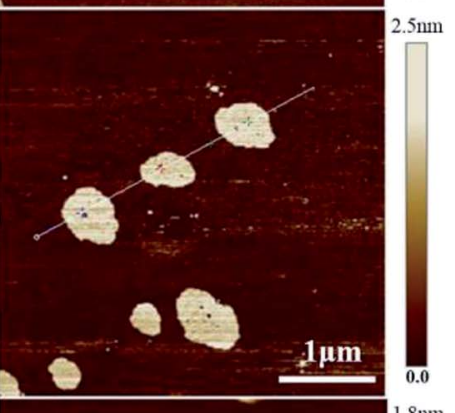

(c)

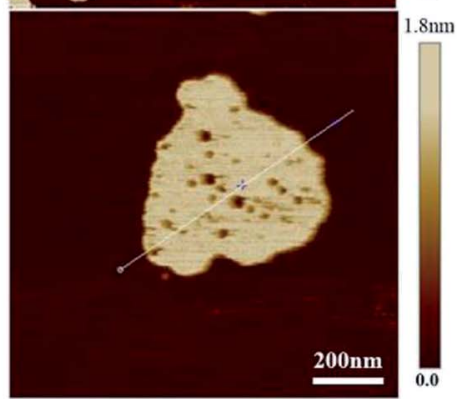

(d)

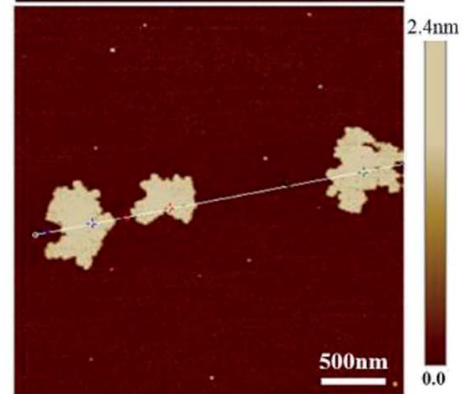

[II]
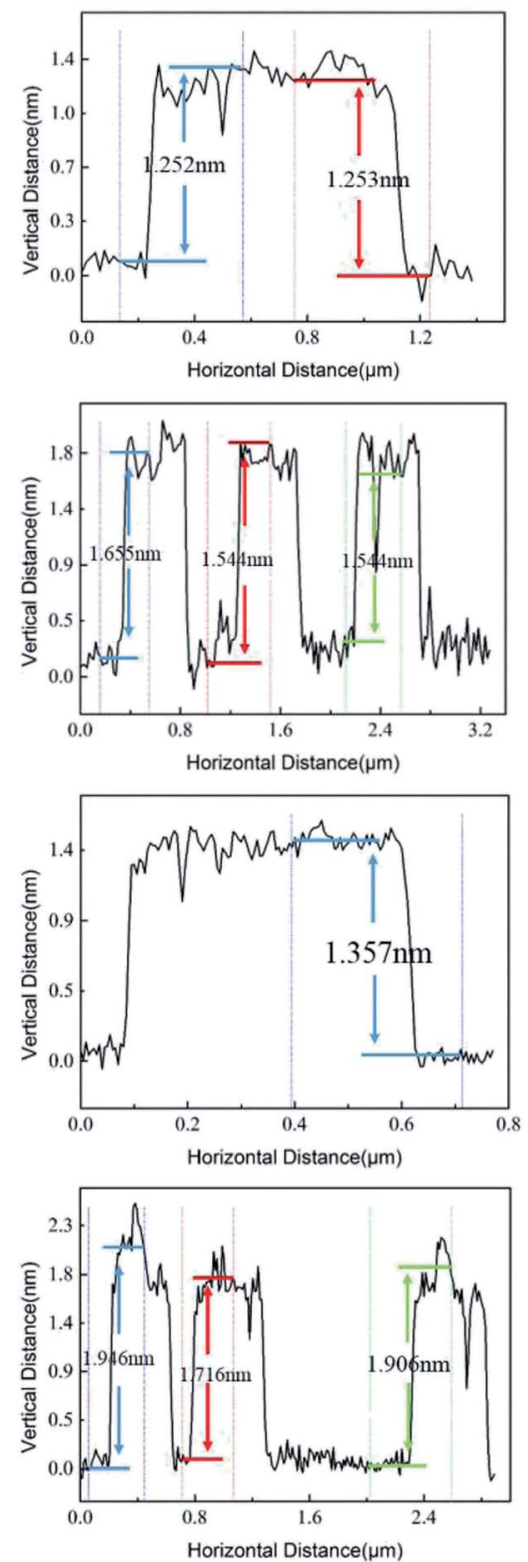

[III]
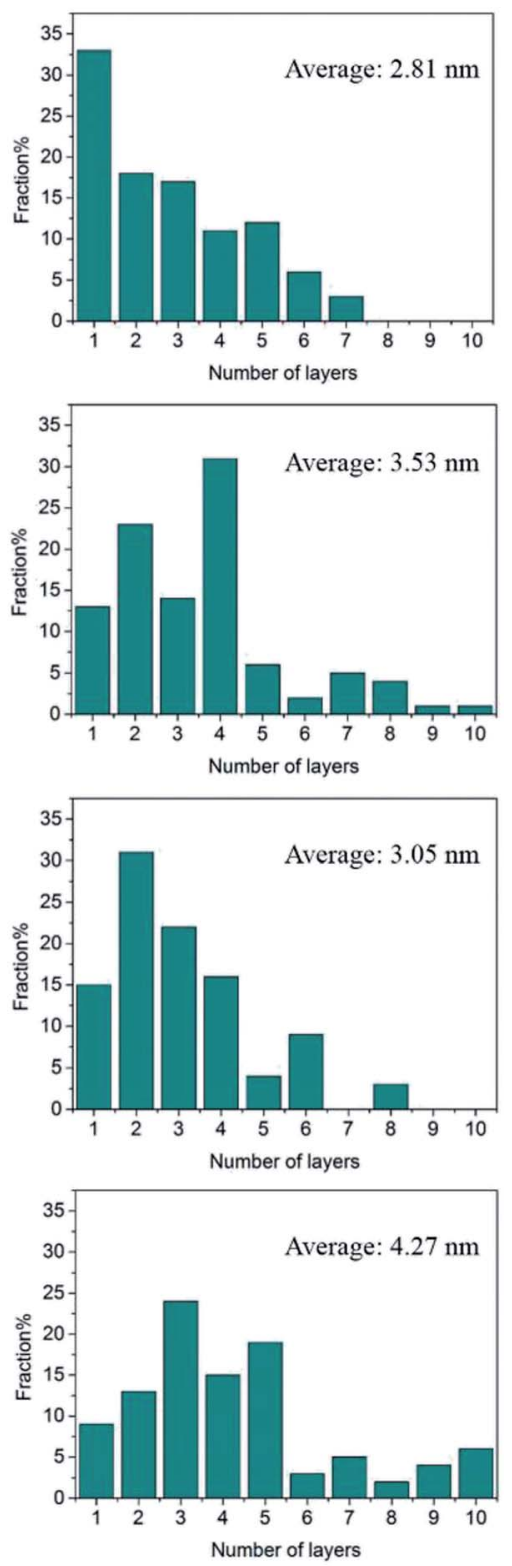

Fig. 4 AFM measurements of the graphene sheets on mica showing morphologies [I], height profiles [II], and a histogram for statistics of layer thickness for about 100 graphene sheets [III] at preparation condition: $0.1 \mathrm{~mol} \mathrm{~L}^{-1}$ (M) $\mathrm{K}_{2} \mathrm{SO}_{4}(\mathrm{a}), 0.2 \mathrm{M}$ (b), $0.3 \mathrm{M}$ (c), $0.4 \mathrm{M}$ (d).

interlocking of individual EGs. In the GP, the bonding between EGs are the crosslinks, which include van der Waals interaction and hydrogen bond. ${ }^{23}$ Fig. 5b sketches the tension-shear transition mechanism in the uniaxial in-plane tension experiments. When tension loads onto the hierarchical structure, the tension stress will transform into the shear stress according to DTS model. Graphene-based paper materials are assembled by EGs in a layer-by-layer manner. Because of the wrinkle morphology of EGs, the interlayer distance is about $1.2 \mathrm{~nm} .{ }^{24}$ which is much larger than the intra-layer distance of EGs $(0.35 \mathrm{~nm})$. The intra-layer binding strength is about two orders higher than that of interlayer according to the van der Waals force equation, which is the function of inter- and intra-layer distances. ${ }^{25}$ Thus the fracture of GP strips should be the separation of interlayers while not the intra-layers of EGs. And the interlayer crosslinks are the binding characterized by the fracture strength. Fig. 5c shows the relationship between the fracture strength and the thickness of EGs. As shown by Fig. 5c, the fracture strength decreases with the thickness increase of EGs. This should be attribute to the decrease of interlayer crosslinks because quantity of interlayer crosslinks will decrease with the thickness increase of individual EGs. 


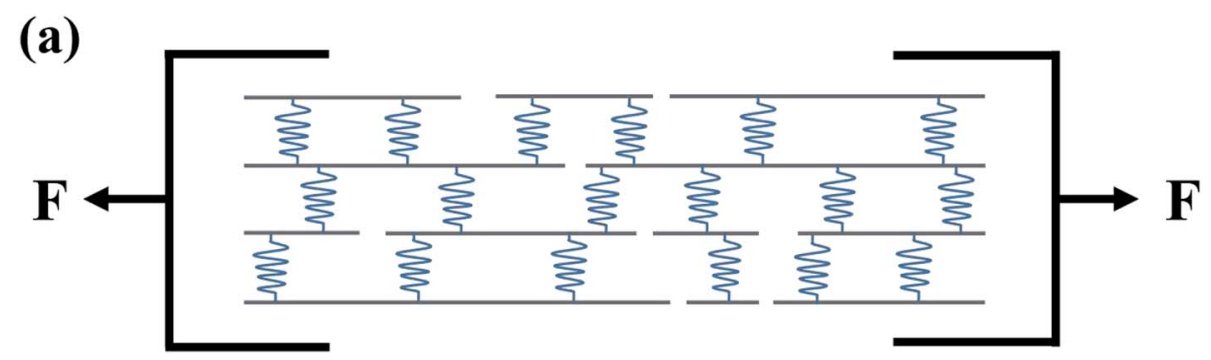

(b)

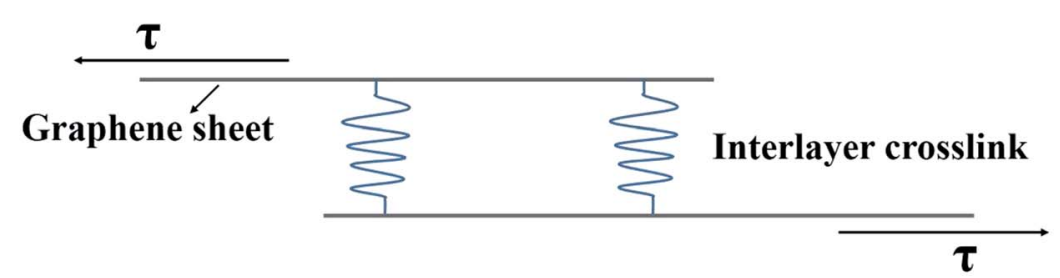

(c)

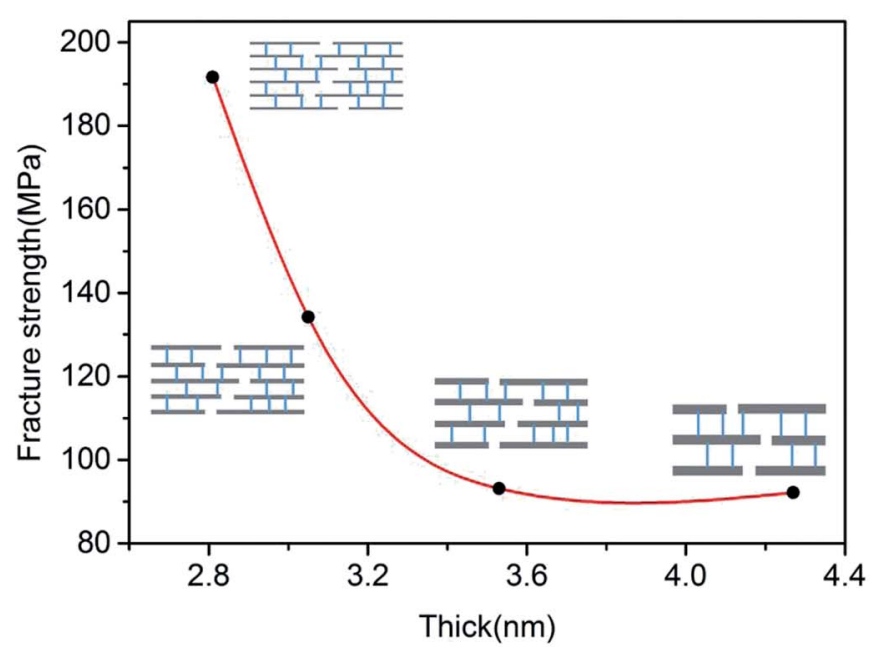

Fig. 5 (a) Schematic drawings of the uniaxial in-plane load-to-fracture of GP strips. (b) A schematic illustration of tension-shear transition, and how the interlayer crosslinks hinder the shear stress. (c) System diagram of graphene sheets thickness and fracture strength.

\section{Conclusions}

In this work, we introduced a route to fabricate graphene sheets by electrochemical exfoliation of graphite foils in $\mathrm{K}_{2} \mathrm{SO}_{4}$ solution. We found the thickness of graphene sheets has strong impact on the fracture strength of corresponding GPs. Combining with the stratified structure of GPs (interlocking of EGs) revealed by SEM observation and the DTS model, we proposed that decreasing the thickness of EGs will increase the number of interlayer crosslinks of GPs, thus enhancing fracture strength of GPs.

\section{Conflicts of interest}

There are no conflicts to declare.

\section{Acknowledgements}

This work was supported by funding from the NSFC (21676005), the NSFCDFG joint project (51761135129), Beijing Municipal
Natural Science Foundation (2172002), and Great Wall Scholarship Project (CIT\&TCD20170306).

\section{References}

1 X. Wang and G. Shi, Energy Environ. Sci., 2015, 8(3), 790-823. 2 M. Z. B. Dzukarnain, T. Takami, H. Imai and T. Ogino, Thin Solid Films, 2016, 615, 247-255.

3 M. J. Allen, V. C. Tung and R. B. Kaner, Chem. Rev., 2010, $110(1), 132$.

4 K. S. Novoselov, A. K. Geim, S. V. Morozov, D. Jiang, Y. Zhang, S. V. Dubonos, I. V. Grigorieva and A. A. Firsov, Science, 2004, 306(5696), 666-669.

5 D. Lin, Y. Liu, Z. Liang, H. W. Lee, J. Sun, H. Wang, K. Yan, J. Xie and Y. Cui, Nat. Nanotechnol., 2016, 11(7), 626.

6 G. Wang, X. Sun, F. Lu, H. Sun, M. Yu, W. Jiang, C. Liu and J. Lian, Small, 2012, 8(3), 452-459.

7 F. Xiao, Y. Li, X. Zan, K. Liao, R. Xu and H. Duan, Adv. Funct. Mater., 2012, 22(12), 2487-2494. 
8 H. Liu, H. Wang and X. Zhang, Adv. Mater., 2015, 27(2), 249254.

9 D. A. Dikin, E. J. Zimney, S. Stankovich, R. D. Piner, G. H. B. Dommett, and R. S. Ruoff, APS March Meeting, American Physical Society, 2007.

10 K. W. Kim, J. H. Kim, S. Cho, K. Shin and S. H. Kim, Thin Solid Films, 2017, 632, 50-54.

11 H. L. Cox, Br. J. Appl. Phys., 1951, 3(3), 72.

12 X. L. Gao and K. Li, Int. J. Solids Struct., 2005, 42(5), 16491667.

13 B. Ji and H. Gao, J. Mech. Phys. Solids, 2004, 52(9), 1963-1990.

14 F. Barthelat, H. Tang, P. D. Zavattieri, C. M. Li and H. D. Espinosa, J. Mech. Phys. Solids, 2007, 55(2), 306-337.

15 Y. Liu, B. Xie, Z. Zhang, Q. Zheng and Z. Xu, J. Mech. Phys. Solids, 2012, 60(4), 591-605.

16 J. Wang, Y. Zhang, J. Ma, J. Li and Z. Zhang, Acta Metall. Sin., 2017, 53(12), 1627-1635.

17 H. Chen, M. B. Müller, K. J. Gilmore, G. G. Wallace and D. Li, Adv. Mater., 2010, 20(18), 3557-3561.
18 D. A. Dikin, S. Stankovich, E. J. Zimney, R. D. Piner, G. H. B. Dommett, G. Evmenenko, S. B. T. Nguyen and R. S. Ruoff, Nature, 2007, 448(7152), 457-460.

19 K. Parvez, Z. S. Wu, R. Li, X. Liu, R. Graf, X. Feng and K. Müllen, J. Am. Chem. Soc., 2014, 136(16), 6083-6091.

20 A. R. Ranjbartoreh, B. Wang, X. Shen and G. Wang, J. Appl. Phys., 2011, 109(1), 666-668.

21 Z. Cheng, Q. Zhou, C. Wang, Q. Li, C. Wang and Y. Fang, Nano Lett., 2011, 11(2), 767-771.

22 A. Carpinteri and N. M. Pugno, Int. J. Fract., 2008, 150(1-2), 221-226.

23 A. Lerf, A. Buchateiner, J. Pieper, S. Schöttl, I. Dekany, T. Szabo and H. P. Boehm, J. Phys. Chem. Solids, 2006, 67(5-6), 1106-1110.

24 Y. Gao, L. Q. Liu, S. Z. Zu, K. Peng, D. Zhou, B. H. Han and Z. Zhang, ACS Nano, 2011, 5(3), 2134-2141.

25 L. Spanu, S. Sorella and G. Galli, Phys. Rev. Lett., 2009, 103(19), 196401. 